\title{
Cellulose Langmuir-Blodgett Films for Moisture and Gaseous Molecular Sensing System
}

\author{
Hiroyuki Kusano, Shin-ichi Kimura and Masahiko Kitagawa
}

Additional information is available at the end of the chapter

http://dx.doi.org/10.5772/50528

\section{Introduction}

Cellulose is not only the main constituent of cell walls of a variety of plants but also one of the most important and versatile renewable resources in the world [1]. The cellulose derivative shows high sensitivity to moisture owing to its hydrophilic character on the glucose base unit and therefore a modification on side chains can act itself as ambipolar adsorber for ambient gas molecules. Thus, the well-defined and tailored cellulose film is a crucial step for the realization of gas sensitive polymer film dedicated for various applications, such as food gas sensitive unit based on the quartz crystal oscillator (QCO).

Langmuir - Blodgett (LB) method is well-established as the mono-molecular deposition technique in which the molecules exhibits well oriented and layered structures with a controllable manner, thus the LB based film is characterized as a film with the mono-layer designed function. Small molecular based LB films have disadvantages in terms of the stability and in-plane orientation, that is, molecular ordering in the plane of monolayer films or substrates, although they are known to form excellent films having periodic molecular arrays along the film deposition direction $[2,3]$.

Cellulose LB films, on the other hand, can overcome these problems, because of the inherent rigidity of the cellulose backbone, as well as the strictly designable molecular structure with reactive hydroxyl groups in glucose unit [4-7] and, therefore, the LB-cellulose film is expected to show the higher stability than that of the cast film because of the higher regularity and rigidity of the LB-stacked film $[8,9]$.

In this chapter, we review concisely the understanding and application of cellulose and thin film; especially LB thin film advancement, gas adsorption on cellulose molecular field with special interest in water molecule, merit and necessity of QCO measurement and stability and further applicability as an ethanol detecting technique. We also describe our results of 
characterization for the cellulose LB films, the QCO sensor systems and the characteristics of the cellulose LB QCO sensor.

\section{History of cellulose LB films}

In this section, the history of cellulose LB films is summarized. It is importance is to develop the effective highly tailored cellulose LB sensing film unit for future application.

In the 1980s, the successful application of the LB technique to polymeric systems including cellulose polymer was started. M. Roman has summarized the history of cellulose LB films in his recent extensive review [1]. Here, we have cited the heart of his review since the core importance is to develop the effective highly tailored cellulose LB sensing film unit for future application. He has classified the cellulose LB films into simple three model categories as follows;

\section{Cellulose-Derivative Model Surfaces}

T. Kawaguchi et al. and T. Itoh et al. have elucidated the molecular arrangement in the cellulose-derivative LB films [10,11]. F. Nakatsubo and co-workers have investigated the incorporation of chromophores into the films [12]. M.Tanaka et al. and we have developed the (bio)technological applications of the films $[8,9,13]$. K. Gotoh studied the detergency of a model aqueous detergent solution with respect to arachidic acid LB-layers on spin-coated cellulose acetate films using a quartz crystal microbalance [14].

\section{Regenerated-Cellulose Model Surfaces}

M. Schaub et al. investigated the use of the LB technique to deposit mono- and multilayers of the regenerated-cellulose films onto various substrates. By subsequently cleaving off the trimethylsilyl groups in humid $\mathrm{HCl}$ vapor, the cellulose molecular structure could be regenerated, yielding very smooth cellulose films. The thickness of these films was directly proportional to the number of monolayers $[15,16]$.

F.F. Rossetti et al. elucidated and compared the nano-scale structures of regenerated cellulose surfaces prepared by the LB technique and by spin coating. The authors used noncontact AFM (NC- AFM) and grazing incidence small-angle x-ray scattering (GI-SAXS) to measure the characteristic in-plane length scales, as well as x-ray specular reflectometry to obtain information in the direction normal to the surface. Swelling experiments showed that significant swelling of the model surfaces occurred at relative humidities above $97 \%$ and that, especially in the thicker films, swelling was accompanied by an out-of-plane rearrangement of the cellulose bundles [17].

\section{Native-Cellulose Model Surfaces}

Regenerated cellulose LB films are prepared either cellulose solution in a suitable solvent system or by converting a thin film of a cellulose derivative through chemical reaction into a cellulose film. In either case, the native cellulose morphology is lost in the process and the 
thin cellulose film have a non-native morphology. Native-cellulose LB films are prepared from aqueous colloidal suspensions of nano-scale cellulose fibrils or fragment thereof.

We selected cellulose ester as the cellulose derivative for forming cellulose LB films, which is adopted to a humidity and/or alcoholic molecular sensor. The Cellulose ester has hydrophobic alkyl side chains and hydrophilic glucose unit in polymer back-bone structure with regularity. Highly oriented molecular closed-packing structure can be obtained in making mono-molecular film between air and water interface by using the cellulose ester. The well-oriented multi-layer of the cellulose ester is useful as the thin and tough membrane to prevent swelling of the film by intercalation of water molecules. Therefore, the cellulose ester LB film is suitable for the moisture and gas sensing systems in comparison with two other models.

Recently, Y. Habibi et al. studied the use of the LB technique to transfer monolayers of cellulose nano-crystals onto silicon wafer substrates and demonstrated that highly oriented cellulose nanocrystal films could also be prepared by solvent casting in an electric field [18].

\section{Characterization of cellulose-derivative LB films}

We illustrate our results of studies on the determination of the surface and bulk properties of the cellulose derivative (palmitoyl cellulose) LB films and confirm the formation of the well-defined solid-like film by using scanning probe microscopy (SPM), Fourier Transforminfrared (FT-IR) absorption spectroscopy, small-angle X-ray diffractometry(XRD) and high resolution transmission electron microscope (HR-TEM) [19-22].

\subsection{Pressure-area isotherm}

Figure 1 shows the surface pressure-area isotherm for the palmitoyl cellulose on the surface of double-distilled water at $290 \mathrm{~K}$ and inset in Figure 1 shows the molecular structure of the palmitoyl cellulose. Method for sample preparation has been described in detail elsewhere 8]. A monolayer of stearic acid was deposited at first on the indium tin oxide (ITO) substrate surface to enhance the contact and then well-defined X-type layers of the palmitoyl cellulose LB film were built up by using the horizontal lifting method at a trough temperature of $290 \mathrm{~K}$. The limiting molecular area for the condensed region at the surface pressure from 5 to $30 \mathrm{mN} / \mathrm{m}$ of palmitoyl cellulose obtained from Fig. 1 was determined to be $60 \AA^{2}$ per glucose unit and the molecular area for condensation was about $44 \AA^{2}$ at $20 \mathrm{mN} / \mathrm{m}$, which confirms the formation of the well-defined solid-like film with the area of about $50 \AA^{2}$. The value is less than that of cellulose esters, 54 to $66 \AA^{2}$ per glucose unit as reported by Kawaguchi et al. [10]. Therefore, it is emphasized that stable the palmitoyl cellulose Langmuir-monolayers were obtained by adjusting the optimum surface pressure during deposition at $20 \mathrm{mN} / \mathrm{m}$. 


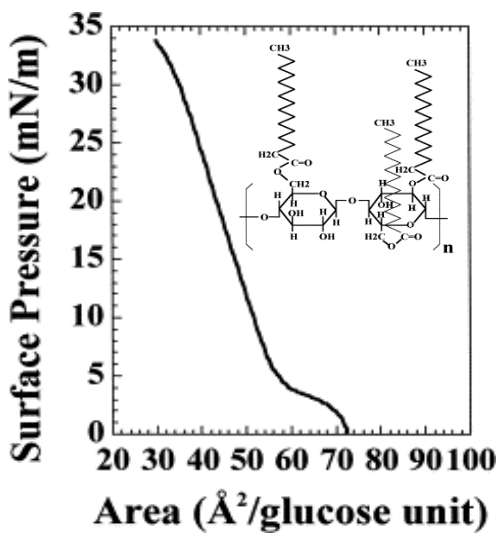

Figure 1. Surface pressure-area isotherm for palmitoyl cellulose at $290 \mathrm{~K}$ (inset: Molecular structure of the palmitoyl cellulose)

\subsection{SPM}

Figure 2 shows the SPM profile of the palmitoyl cellulose 5 layers LB film. SPM profile showed grains with average size of $200 \mathrm{~nm}$ in diameter. The observed height of the grain was about $11 \mathrm{~nm}$ for the five mono-layered LB film. Thus, the mono-layer thickness of $22 \AA$ was estimated, which is consistent with the reported values between 20 and $26 \AA$ [19].

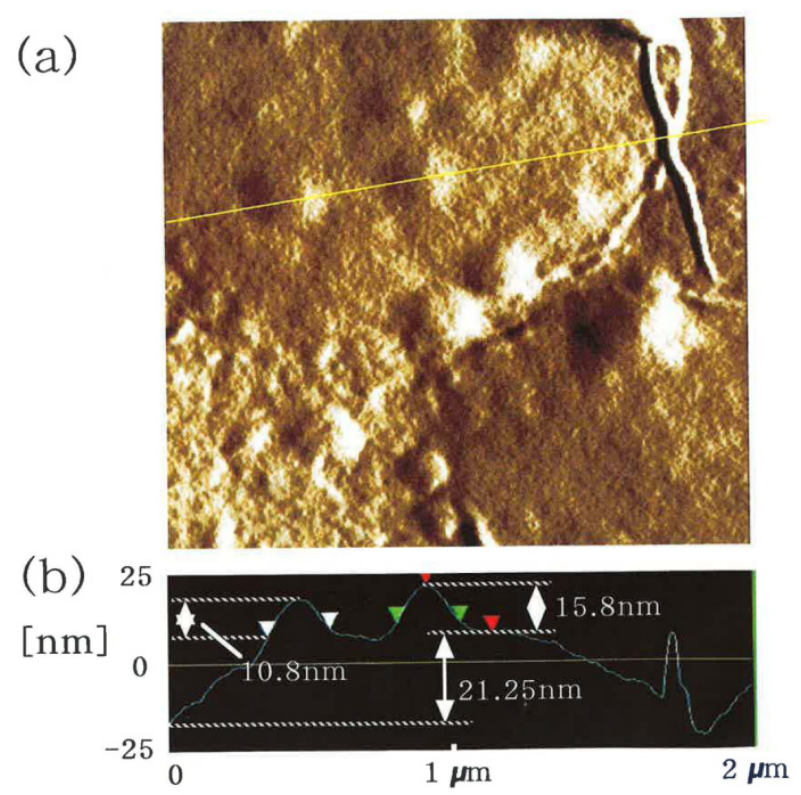

Figure 2. The SPM of palmitoyl cellulose 5 layered LB film. (a)SPM image and (b) SPM section analysis [19]. 


\subsection{FT-IR}

Figure 3(a) and (b) show expanded infrared spectra for the palmitoyl cellulose between 1500 $\mathrm{cm}^{-1}$ and $3000 \mathrm{~cm}^{-1}$, for the LB films and the cast films [19]. In the FT-IR RAS measurement, p-polarized electric field was used, where the electric field vector of the incident light excites selectively the IR mode which has the molecular vibration perpendicular to the film surface, i.e., to the substrate surface. Hence, in the case of the well-defined palmitoyl cellulose LB film, the $\mathrm{CH}_{2}$ asymmetric mode which has a stretching direction perpendicular to the substrate can be excited selectively compared with the $\mathrm{CO}$ mode which has a stretching direction parallel to the substrate. On the other hand, on the film that has been cast, each palmitoyl cellulose takes a random orientation with respect to the substrate surface. As a result, the orientation of $\mathrm{CH}_{2}$ bonds and $\mathrm{CO}$ bonds are random. Therefore, intensities of $\mathrm{CO}$ vibration modes decrease in the LB films compared with the cast films. In this case, the ratio $\mathrm{Im} / \mathrm{Ik}$ of LB film was 0.45 , where $\mathrm{Im}$ is the intensity for the $\mathrm{CH}_{2}$ asymmetric mode and $\mathrm{Ik}$ is the intensity for the $\mathrm{C}=\mathrm{O}$ mode. The ratio for the cast film was 0.20 . These results confirm the formation of the mono-layered LB film.

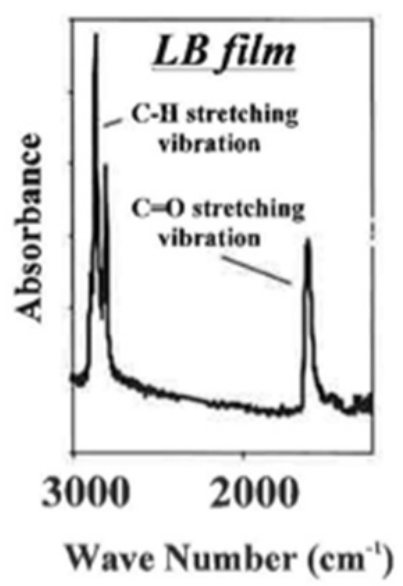

(a)

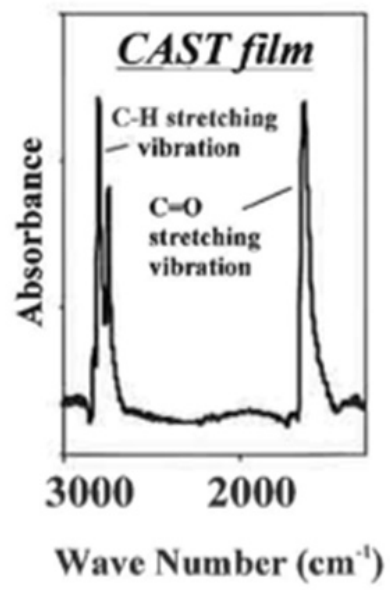

(b)

Figure 3. IR absorption between $1500 \mathrm{~cm}^{-1}$ and $3000 \mathrm{~cm}^{-1}$ for the palmitoyl cellulose films (a) LB film and (b) cast film.

\subsection{XRD}

Figure 4 shows In-plane X-ray diffraction pattern of the PC-LB film on the ITO substrate. The In-plane X-ray diffraction pattern were recorded on an X-ray diffractometer equipped with a 4 -axes goniometer. Cuk $\alpha$ radiation from copper rotating anode was used for the experiment. Incident angle $(\omega)$ for the in-plane geometry was between $0.14^{\circ}$ and $0.36^{\circ}$. Diffraction angle of In-plane geometry is defined as $2 \theta \chi$. The peak intensity of $2 \theta \chi=21.1^{\circ}$ (peak A) at $\omega=0.14^{\circ}$ was larger than that of at $\omega=0.20^{\circ}$. To the contrary, the peaks of 
$2 \theta \chi=30.7^{\circ}$ (peak B), $35.5^{\circ}$ (peak C) appeared at $\omega=0.20^{\circ}$ almost disappeared at $\omega=0.14^{\circ}$ [21]. The interplanar spacings of these two peaks $(B, C)$ correspond to those of ITO(222), $\mathrm{ITO}(004)$.

The peak intensity of A decreased with the increase of intent angle. Contrary, the peak intensities of ITO substrate increased with increase incident angle. Consequently, the peak A is due to the diffraction peak of the PC-LB film, which appeared at the small incident angle of X-ray $\left(\omega=0.14^{\circ}\right)$. The interplanar spacing of the peak A of the PC-LB film was $4.20 \AA$. This value was attributed to a distance between the palmitoyl chains in the PC molecules perpendicular to the glucose unit. Palmitoyl chains were long acyl group (16 carbon) and bonded to the hydroxyl group of the glucose unit as shown in Fig.1.

On the other hands, the size of apparent crystallite can be estimated from the peak broadening. Hence, the size of crystallite can be estimated by the Scherrer equation $(\mathrm{D}=$ $0.9 \lambda /(\beta \cos \theta))$, where $\mathrm{D}$ is the size of crystallite, $\lambda$ is wave length of $\mathrm{CuK} \alpha, \beta$ is the half width of the peak and $\theta$ is the Bragg angle. From the half width of the peak of $2 \theta \chi=21.1^{\circ}$, the size apparent of crystallite in the PC-LB film was about $15 \AA$.

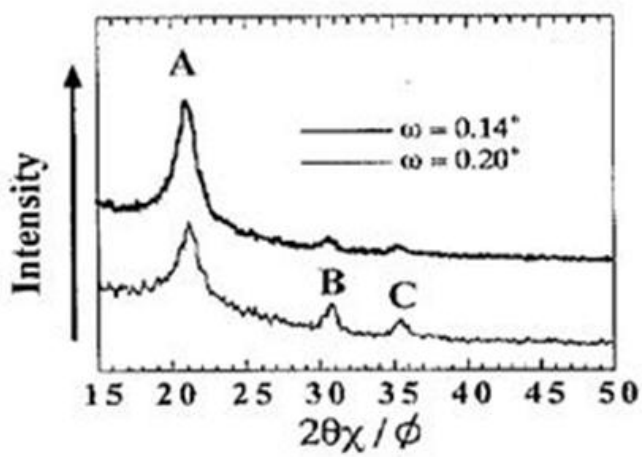

Figure 4. In-plane X-ray diffraction pattern of palmitoyl cellulose Langmuir-Blodgett film on the ITO substrate.

\subsection{HR-TEM}

Figure 5 is an example of a high resolution TEM image of the surface of Pt coated LB film condensed at $293 \mathrm{~K}$ under the surface pressure of $20 \mathrm{mNm}^{-1}$ [20]. Grains with larger size than $200 \mathrm{~nm}$ in diameter have been observed.

Figure 6 shows a schematic model of the PC-LB film on the ITO substrate. The structure of the PC-LB film is regular in the direction parallel to the film substrate and the distance between the palmitoyl chains keep some same distance. The conformation of palmitoyl chains is most probably determined due to the cellulose main chains.

Figure 7 illustrates the model of grains in the PC-LB film. We already described the grain size was estimated $200 \mathrm{~nm}$ by using SPM and TEM. Therefore, the structure of $200 \mathrm{~nm}$ size 
grains in the PC-LB film is considered to be aggregation of many $15 \AA$ size crystallites with $4.20 \AA$ A distance between the palmitoyl chains.

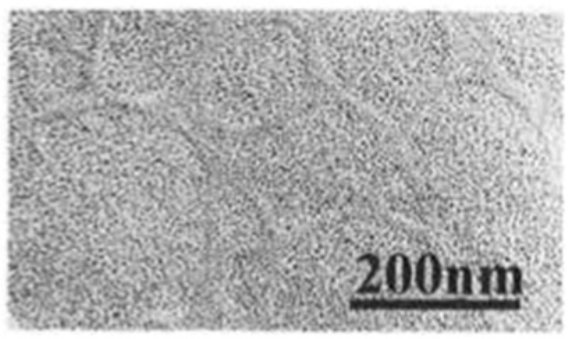

Figure 5. TEM image of the palmitoyl cellulose LB films deposited at 293K. Circle areas are palmitoyl cellulose grains.

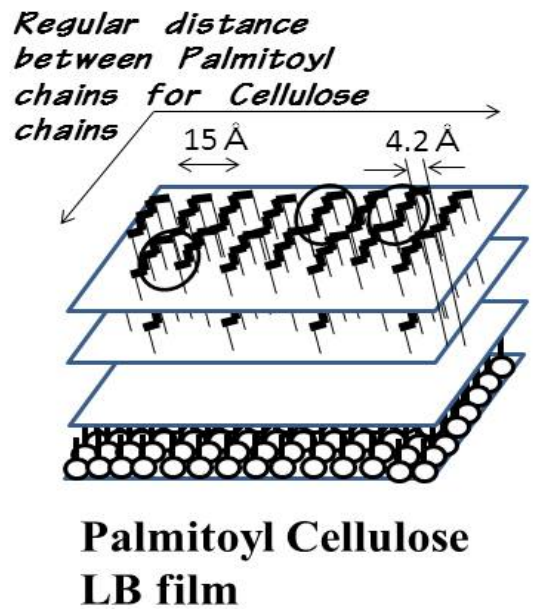

Figure 6. Schematic model of the PC-LB film on the ITO substrate.
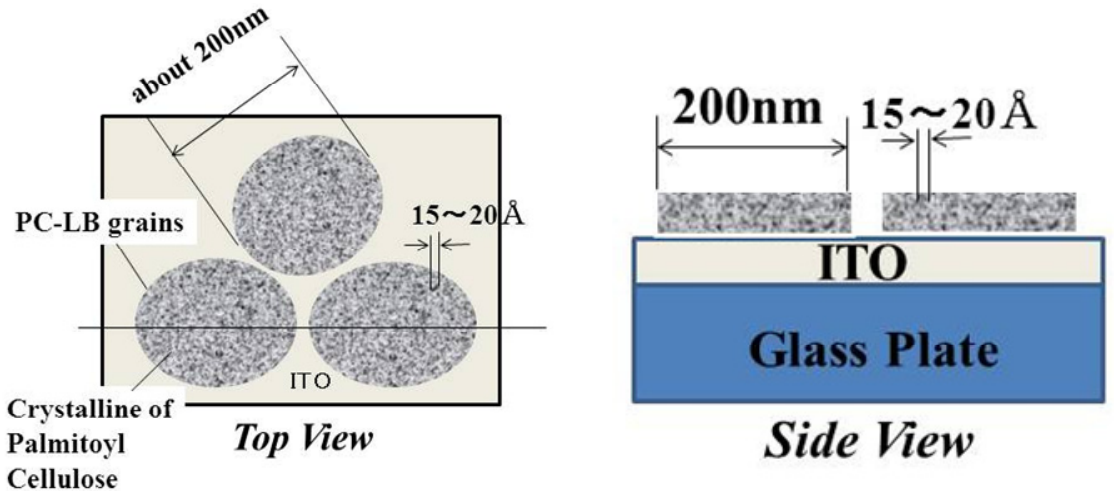

Figure 7. The model of grains in the PC-LB film. 


\section{Cellulose-derivative LB film based humidity sensing system}

We show our study of the humidity measurement system using the QCO coated with the cellulose LB film [8,9]. We clarified the water absorption and desorption mechanism for the cellulose-derivative LB films. The dependence of the sorption properties on the number of layers clearly indicated that the two regions which hold in row and high humidity condition show different sorption characteristics, and thus the evidence showed that the modes of sorption must be different. Therefore, as the main conclusion of LB cellulose sensing study, the analysis based on the combination of the two sorption models was prefered. The stability test by soaking the sensors in water showed the superiority of LB based sensingfilms with the structurally ordered cellulose molecules.

\subsection{Quartz crystal oscillator measurement system}

Figure 8 illustrates schematic diagram of the quartz crystal oscillator (QCO) coated with the cellulose LB film. A monolayer of stearic acid was deposited on the Au electrode to contact with the hydrophilic head of the stearic acid in order to build up good X-type layers of the palmitoyl cellulose LB films. The horizontal lifting method was used to prepare the X-type LB films on both sides of the QC.

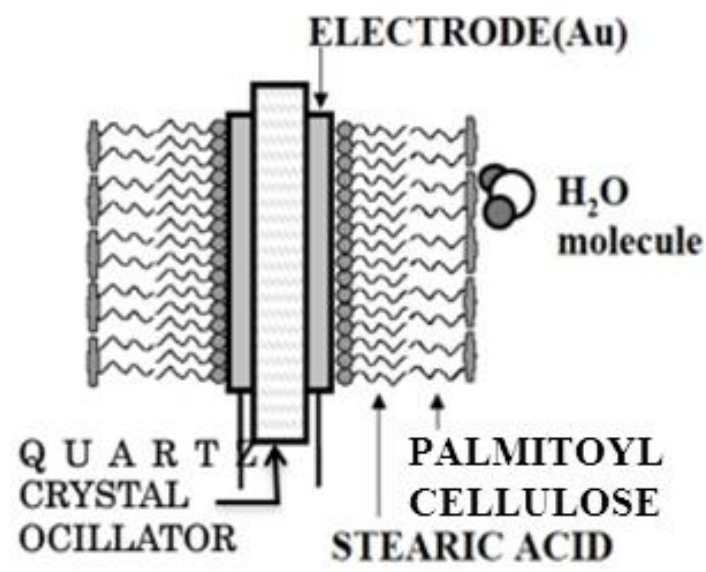

Figure 8. Schematic diagram of the quartz crystal oscillator (QCO) coated with the cellulose LB film.

The humidity measurement system using the quartz crystal oscillator (QCO) coated with the cellulose LB film was constructed with four portions; A:Quartz crystal oscillator (QCO) coated with cellulose LB films; B:Resonant circuit for conversion of QCO mechanical vibration to alternating electrical signal; C:Frequency counter for changing the alternating current signal to numerical data; D:Personal computer for stacking the numerical data.

The frequency of vibration in the QCO with the cellulose LB film changes by the amount of water molecules adsorbed on the surface. Resonating circuit converts the mechanical 
vibration of the QCO into electrical signal. Frequency of the electrical signals is counted and converted into numerical digital signal to input computer for stacking the data and calculation of the value of humidity. The Colpitts resonating electrical circuit was used for the QCO in this study. Inverted amplifier with CMOS IC was high speed enough for quick response of the high frequency (about $15 \mathrm{MHz}$ ) of the QCO. The wave form of the output signals was monitored by the digital oscilloscope (model 54200A, Hewlett Packard Co.). The frequency was corrected with a digital counter (model SC-7202, Toyo Technica Co.). The humidity dependence of the frequency change of the quartz crystal with the cellulose LB films was measured in the desiccator held at a fixed relative humidity. Saturated salt solutions were used to control the humidity in the desiccators.

\section{Adsorption characteristics}

Figure 9(a) shows dependence of the number of sorbed water molecules on the relative humidity for a 4 monolayer LB films. A and B regions in which the temporal dependence of frequency variations with decreasing relative humidity were measured. Figure 9(b) shows desorption characteristics of sorbed water on the LB films, showing the temporal dependence of the frequency changes with decreasing relative humidity in regions $\mathrm{A}$ and $\mathrm{B}$ in Fig. 9, respectively.

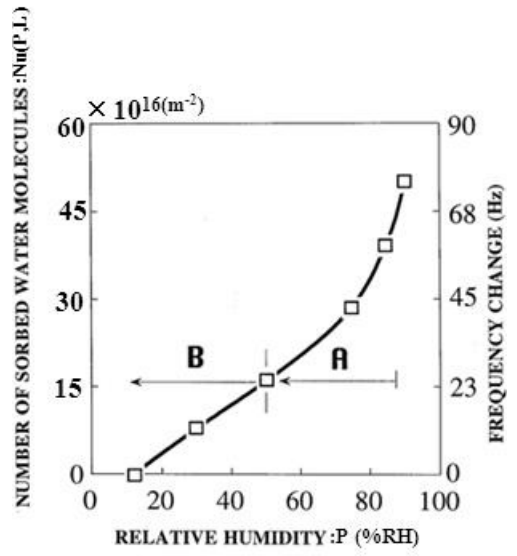

(a)

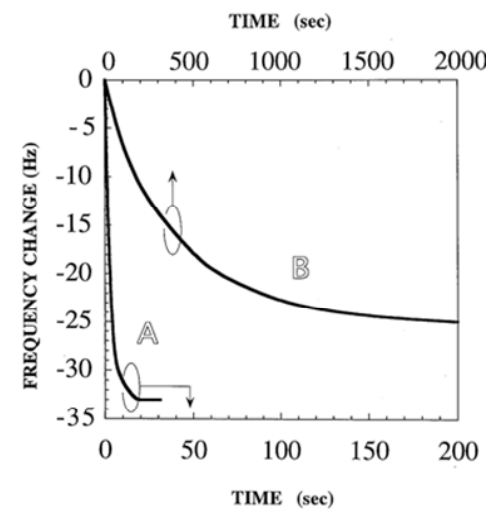

(b)

Figure 9. (a) Dependence of the number of sorbed water molecules on the relative humidity for a 4 monolayer LB films. A and B regions in which the temporal dependence of frequency variations with decreasing relative humidity were measured. (b) Desorption characteristics of sorbed water on LB films, showing the temporal dependence of the frequency changes with decreasing relative humidity in regions A and B in Fig. 9, respectively.

From the viewpoint of standard analysis of the sorption characteristics, Brunauer, Emmett and Teller (BET) analysis should be applied to each data set with an appropriate set of parameters. In the LB cellulose sensor case, the sorption process as an intercalation-like 
molecular process was inevitable. Therefore, the new model in the analysis of sorption properties of the LB monolayered humidity sensors was tested. First, the dependence of the sorption properties on the number of layers clearly indicates that the two regions, which hold in row and high humidity condition show different sorption characteristics, and thus the evidence showed that the modes of sorption must be different. Therefore, as the main conclusion of the LB cellulose sensing study, the analysis based on the combination of the two sorption models was prefered. Second, even if the perfection of the monolayer can be improved further, i.e. if the ordering of the monolayers is not so good, the number of monolayers, and thus the number of molecules, makes no difference to the results obtained in the experiment. This indicates the uniqueness of the existence of the observed two sorption processes in ordinary cellulose humidity sensors. Therefore, new formula could be regarded as providing a better description of sorption characteristics in the humidity and/or molecular sensing study.

\subsection{Water adsorption model}

The water molecule sorption characteristics of the cellulose LB monolayered films have been explained on the basis of the QCM method. These characteristics can be divided into two parts. In the low relative humidity region, desorption/sorption was observed to be slow, with a time constant of about $500 \mathrm{~s}$, whereas in the high relative humidity region it was more rapid, with a time constant of less than $10 \mathrm{~s}$. The dependence of the number of sorbed water molecules on the number of monolayers in the films reveals that two distinct mechanisms are involved in the relative humidity range examined, and the number of sorbed water molecules can be described by a combination of the Freundlich-type and the Polanyi-type functions, in the following form [8]:

$$
N_{u}(p, L)=1.5 \times 10^{16}\left(p-p_{a}\right)^{0.87} L+6.0 \times 10^{20}\left(R T \operatorname{In}\left(p_{0} / p\right)\right)^{-1}-3.0 \times 10^{17}
$$

where $N_{u}(p, L)$ is the number of sorbed water molecules per $\mathrm{m}^{2}, p$ is the relative humidity $(\%), p_{a}$ is the relative humidity at the start of the measurements (\%), $p_{0}$ is the relative humidity at saturation (\%), $L$ is the number of layers in the LB film, $R$ is the gas constant $\left(8.314 \mathrm{~J} \mathrm{~K}^{-1} \mathrm{~mol}^{-1}\right)$, and $T$ is the absolute temperature $(\mathrm{K})$.

Figure 10 shows the sorption model (the structure of the palmitoyl cellulose LB film is shown in Fig. 1) including both Freundlich-type and Polanyi-type sorption.

In the low relative humidity region the characteristics can be described by the Freundlich isotherm, and show a linear dependence on the number of monolayers in the film. We interpret this as being due to the sorption of water into the cellulose LB monolayer plane, so that the Freundlich-type sorption mechanism determines the water sorption characteristics that predominate in this region. It is explained as sorption onto the hydrophilic sites in the glucose units in the monolayer. The experimentally determined number of sorbed water molecules is 4.7 per glucose unit at almost saturation, which is close to the average number of 4.4 hydrophilic sites per glucose unit in the sample. 
In the high relative humidity region another mechanism dominates the sorption characteristics, as described by the potential theory of Polanyi. In this case, the amount of sorbed water corresponds to a layer thickness of about three water molecules at a fixed point of almost saturation, $90 \% \mathrm{RH}$. In this humidity region the sorption process is controlled by the free condensation of water molecules onto the film surface. The average number of sorbed water molecules per glucose unit calculated using the occupied area of the glucose unit and the water molecule is about 4.4, comparable to the experimentally determined value of about 4.7. These values coincide with the number of base sites not substituted by palmitoyl chloride. The number of hydrophilic sites, such as $-\mathrm{OH}$ and $-\mathrm{C}=\mathrm{O}$, per glucose unit in the sample is 4.3. Thus the number of sorbed water molecules is thought to be limited simultaneously by both steric occupancy and site occupancy factors. We have constructed the water sorption mechanism onto the glucose unit of the cellulose polymer LB film as a measure of gas molecular sorption phenomena [13].
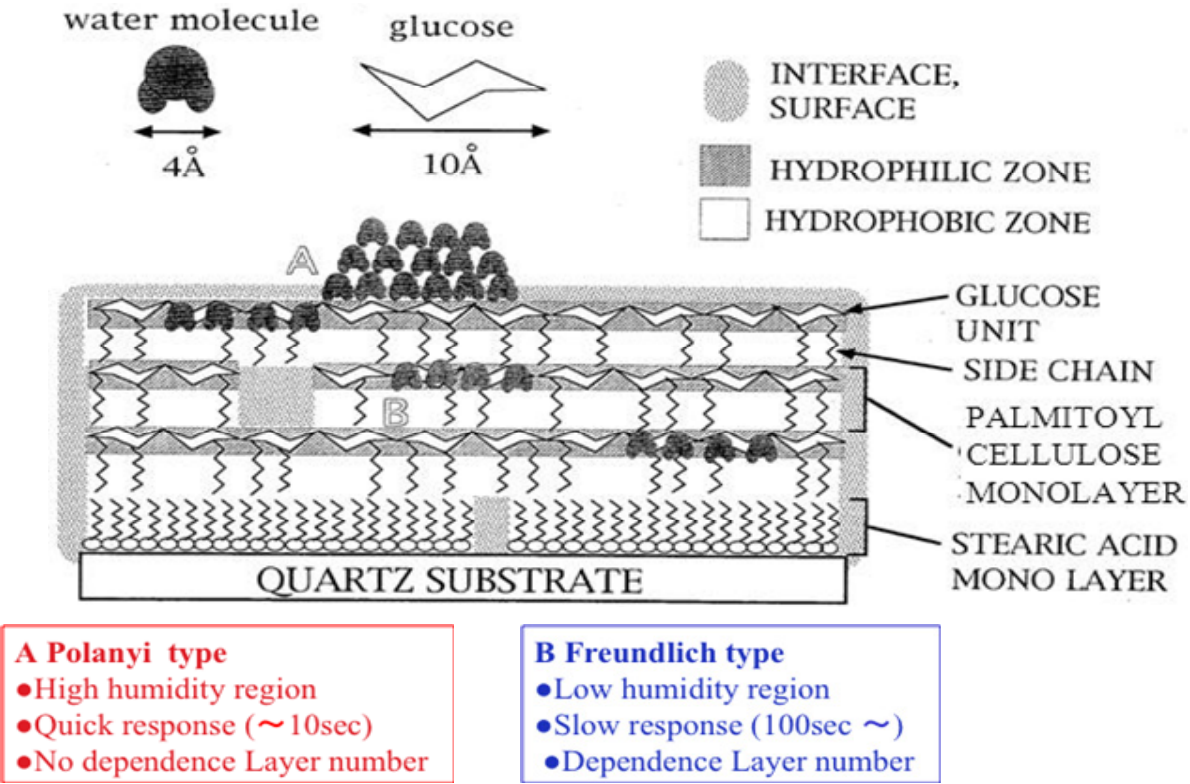

B Freundlich type

- Low humidity region

- Slow response $(100 \mathrm{sec} \sim)$

$\bullet$ Dependence Layer number

Figure 10. Schematic diagram of the proposed mechanism of water sorption in palmitoyl cellulose polymer LB film [13].

\subsection{Durability test}

Figure 11 shows durability of the LB-based QCO sensor against water. $X$ is the amount of the cellulose molecules after soaking the QCO's into the water and then drying, $X_{0}$ is the initial amount reading at the measurement condition at $80 \% \mathrm{RH}$. We have then tested both 
the cellulose LB-QCO sensors and the cast cellulose bulk QCO sensors in the sequence of simulated high humidity condition by soaking sensors into the water bath at several cycles, as the durability test of the LB-cellulose based QCO sensor and the cast cellulose QCO sensor against water, since a major issue with gas sensors is their unstability to humidity because of today's requirement of stable measurement at high humidity for the use of agricultural factory in the near-saturation condition of water vapor.

The test condition of stability against water was defined to soak the sensors in water for 30 minutes at room temperature between the measurements. The stability was defined as the change rate, that is, the ratio of frequency change normalized by the initial value in the measurement of humidity at the condition of $80 \% \mathrm{RH}$ and $30^{\circ} \mathrm{C}$. The indicated value of humidity for the cast type cellulose sensor showed steep decrease in signal read out with increasing cycle of test measurement after each soaking. On the other hand, the LB-based QCO sensor did show no change in read out at all in the frequency change.

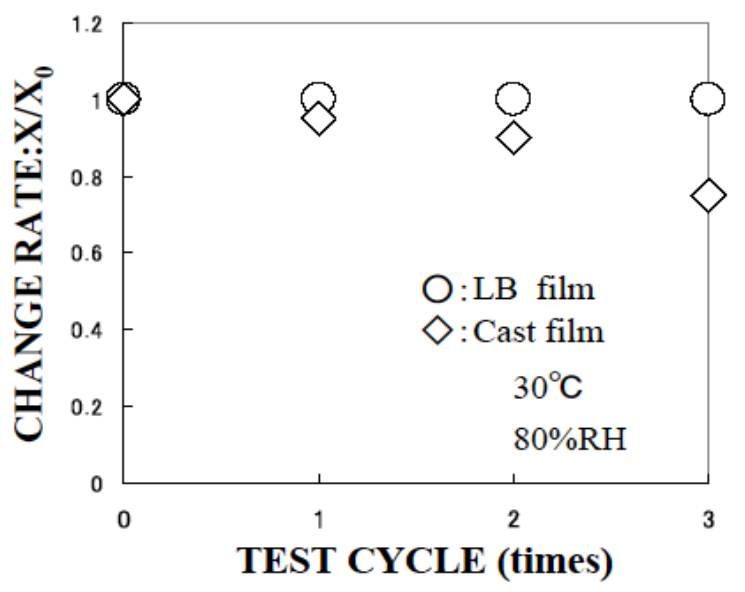

Figure 11. Durability of the LB-based QCO sensor against water. $X$ is the amount of the cellulose molecules after soaking the QCO's into the water and then drying, $\mathrm{X}_{0}$ is the initial amount reading at the measurement condition at $80 \% \mathrm{RH}[9]$.

\section{Cellulose-derivative LB film based alcohol sensing system}

We describe our recent results of the adsorption characteristics of ethanol gas and the possibility of development of the sensitive and stable gaseous molecular-sensing system by the advanced QCO based LB cellulose sensing system [9]. 


\subsection{Ethyl alcohol adsorption}

Specific gaseous molecules such as ethanol have been tested. After a steady frequency was attained in a dry $\mathrm{N}_{2}$ atmosphere and in an air atmosphere, the time-dependence of the frequency change of the LB-based QCO sensor was recorded while exposing to gaseous alcohols (Ethyl alcohol, Isopropyl alcohol and so on.).

Figure 12 shows temporal response of the frequency change of the LB-based QCO sensor exposing to gaseous Ethyl alcohol. The definite and reproducible sensor responses for these gaseous alcohols have been obtained in the temporal response of the frequency change of the LB-based QCO sensor exposed to evaporated Ethyl alcohol. Under the ethanol adsorption and desorption condition, the frequency changes with delay times of about 30s were observed and the response time was an order of magnitude shorter than that of water. Similar to the water adsorption, alcohol adsorption on molecular films seems to be able to be well explained and estimated on the basis of hydrophilicity of the LB cellulose films, as we have already established to explain the adsorption of water molecules on molecularly-welloriented and structured LB film surface. Therefore, development of sensitive and stable moisture and gaseous molecular-sensing and discrimination system for alcoholic beverages gases is thus possible by the advanced QCO based LB cellulose sensing system.

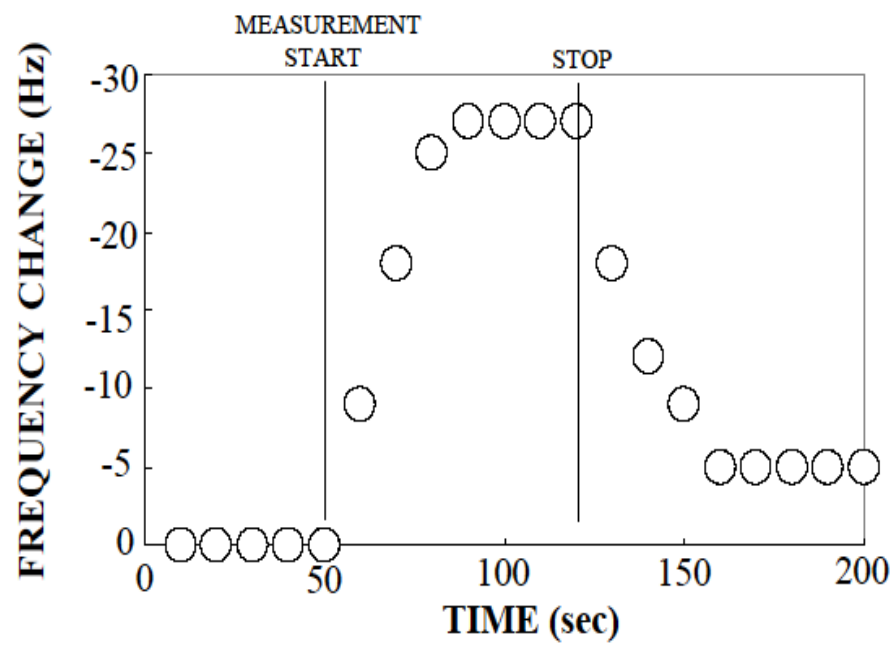

Figure 12. Temporal response of the frequency change of the LB-based QCO sensor exposing to gaseous Ethyl alcohol [9]. 


\section{Perspectives}

We have reviewed the studies and the advances of the cellulose Langmuir-Blodgett (LB) films and the cellulose LB based quartz crystal oscillator $(\mathrm{QCO})$ sensors.

We described our results of characterization for the cellulose LB films by using scanning probe microscopy (SPM), Fourier Transform-infrared (FT-IR) absorption spectroscopy, small-angle X-ray diffractometry (XRD) and high resolution transmission electron microscope (HR-TEM).

We also described concisely the understanding and application of the cellulose and thin film; especially LB thin film advancement, gas adsorption on cellulose molecular field with special interest in water molecule, merit and necessity of QCO measurement and stability and further applicability as an ethanol detecting technique.

One of the most important points in developing alcoholic gas sensing is to realize simple discrimination between ethanol and water. We have examined the ethanol gas sensors by using the cellulose LB QCO. Similar to the water case, ethanol adsorption on molecular films seems to be able to be well defined on the basis of hydrophilicity of the LB cellulose films. Therefore, sensitive and stable moisture and gaseous molecular-sensing and discrimination system for alcoholic beverage gases are promising by the advanced QCO based LB cellulose sensing system in near future.

\section{Author details}

Hiroyuki Kusano and Shin-ichi Kimura

Tottori Institute of Industrial Technology, Wakabadai, Tottori, Japan

Masahiko Kitagawa

Department of Information and Electronics, Graduate School of Engineering, Tottori University, Koyama, Tottori, Japan

\section{Acknowledgement}

We would like to thank Takaaki Hishinuma of Tottori university for his help in the cause of this study.

\section{References}

[1] Roman M (2010) Model Cellulose Surface: History and Recent Advaces. ACS Symposium Series:3-53.

[2] Sugi M, Carter F. L, Siatkowski R. E, Wohltjen H, ed. (1988) Molecular Electronic Devices. Amsterdam, Elsevier. 
[3] Petty M.C (1996) Langmuir-Blodgett films An introduction. Cambridge University Press, $\mathrm{UK}$.

[4] Schaub M, Fakirov C, Schmidt A, Lieser G, Wenz G, Wegner G, Albouy P.-A, Wu H, Foster M.D, Majrkzak C, Satija S, (1995) Macromolecules 28: 1221.

[5] Basque P, Gunzbourg Ad, Rondeau P, A, Ritcey M (1996) Langmuir 12:5614.

[6] Redl F.X, Lutz M, Daub J, (2001) Chem. Eur. J. :75350.

[7] Redl F.X, Kothe O, Rockl K, Bauer W, Daub J (2000) Macromol. Chem. Phys. 201:2091.

[8] Kusano H, Kimura S, Kitagawa M, Kobayashi H (1997) Thin Solid Films 295: 53-59.

[9] Kusano H, Kitagawa M (2008) IEICE TRANS. ELECTRON. E91-C, 12: 1876-1879.

[10] Kawaguchi T, Nakahara H, Fukuda K (1985) Monomolecular and Multimolecular Films of Cellulose Esters with Various Alkyl Chains. Thin Solid Films.133:29-38.

[11] Itoh T, Tsujii Y, Fukuda T, Miyamoto T, Ito S, Asada T, Yamamoto M (1991) Fluorescence Spectroscopy for a Cellulose Trioctadecanoate Monolayer at the AirWater-Interface. Langmuir. 7:2803-2807.

[12] Sakakibara K, Ogawa Y, Nakatsubo (2007) F First cellulose Langmuir- Blodgett films towards photocurrent generation systems. Macromol. Rapid Commun. 28:12701275.

[13] Hillebrandt H, Tanaka M, Sackmann E (2002) A novel membrane charge sensor: Sensitive detection of surface charge at polymer/lipid composite films on indium tin oxide electrodes. J. Phys. Chem. B. 106:477-486.

[14] Gotoh K, Nakata Y, Tagawa M (2006) Evaluation of particle deposition in aqueous solutions by the quartz crystal microbalance method. Colloid Surf. A-Physicochem. Eng. Asp. 272:117-123.

[15] Schaub M, Wenz G, Wegner G, Stein A, Klemm D (1993) Ultrathin Films of Cellulose on Silicon-Wafers. Adv. Mater. 5:919-922.

[16] Buchholz V, Wegner G, Stemme S, Odberg L (1996) Regeneration, derivatization and utilization of cellulose in ultrathin films. Adv. Mater. 8399-402.

[17] Rossetti F. F, Panagiotou P, Schneck F, Dommach M, Funari S.S, Timmann A, MullerBuschbaum P, Tanaka M (2008) Structures of regenerated cellulose films revealed by grazing incidence small-angle $\mathrm{x}$-ray scattering. Biointerphases. 3:117-127.

[18] Habibi Y, Heim T, Douillard R (2008) AC electric field-assisted assembly and alignment of cellulose nanocrystals. J. Polym. Sci. Pt. B-Polym. Phys. 46:1430-1436.

[19] Kimura S, Kusano H, Kitagawa M, Kobayashi H (1999) Layer-by-layer characterization of cellulose Langmuir-Blodgett monolayer films. Appl. Surf. Sci. 142: 585-590.

[20] Kimura S, Kusano H, Kitagawa M, Kobayashi H (1999) TEM characterization of cellulose Langmuir-Blodgett monolayer films. Appl. Surf. Sci., 142: 579-585.

[21] Kimura S, Kitagawa M, Kusano H, Kobayashi H (2000) Electrical and dielectric properties of cellulose LB films. Polym. Adv. Technol. 11:723-726. 
[22] Kimura S, Kitagawa M, Kusano H, Kobayashi H (2001) In and out plane X- ray diffraction of cellulose LB films. In Novel Methods to Study Interfacial Layers; Möbius, D., Miller, R., Eds.; Studies in Interface Science 11; Elsevier: Amsterdam, The Netherlands. 255-264. 